\title{
Evaluation of Real Time PCR for the diagnosis of Extrapulmonary Tuberculosis and comparison with AFB Microscopy among Bangladeshi population
}

\author{
Chowdhury $\mathrm{MR}^{1,2}$, Bari $\mathrm{SMN}^{1}$, Islam $\mathrm{KS}^{3^{*}}$, Rakibuzzaman $\mathrm{AGM}^{1}$ and Afrin $\mathrm{S}^{1}$ \\ 1. Department of Biochemistry and Molecular Biology, University of Dhaka-1000. 2. Gastroliver Hospital and \\ Research Center, Green Road, Panthapath, Dhaka-1205. 3. Department of Biochemistry and Molecular \\ Biology, Jahangirnagar University, Savar, Dhaka-1342.
}

[Received: January 2, Accepted: March 24, 2012]

\begin{abstract}
Tuberculosis is a global public health issue and a disease burden in Africa and Asia. Bangladesh is one of the high burden countries in Southeast Asian region. Mycobacterium tuberculosis the causative agent of tuberculosis, is a devastating bacterium because it spreads person to person, acquired multiple antibiotic resistance and most importantly has the limitation in rapid diagnosis, specially in case of extrapulmonary infection. Here we showed Real-Time PCR assay with the primers targeting IS6110 is a better method than conventional AFB microscopy for the diagnosis of extrapulmonary tuberculosis. For the comparative study 99 extrapulmonary specimens from 9 different parts of the body were collected from suspected patients. Among those only 10 samples were positive, where $50 \%$ them were scanty positive in AFB microscopy, on the other hand 33 samples were positive in Real-Time PCR assay. All samples positive in AFB microscopy were also positive in Real-Time PCR assay with an additional 23 positive. Higher percentage of positive results were found in Real-Time PCR analysis in all the samples except Percutaneous nephrostomy (PCN) fluid and Pus. The sharp differences in the result indicate the effectiveness and of the Real-Time PCR assay over the conventional AFB microscopy. High precision and accuracy make it better choice as a diagnostic method for the diagnosis of extrapulmonary tuberculosis.
\end{abstract}

Key words: AFB microscopy, Extrapulmonary tuberculosis, Mycobacterium tuberculosis, Real-Time PCR.

\section{INTRODUCTION}

Tuberculosis continues to be a major global public health issue. The World Health Organization has estimated an annual incidence of 8.8 million cases of tuberculosis worldwide, with 1.8 million deaths. Most of the disease burden is in Africa and Southeast Asia, where annual incidence rates are 350 and 182 cases per 100,000 populations, respectively ${ }^{[1]}$. The South-East Asia Region, with 4.97 million TB cases, carries over one-third of the global burden of TB. Five of the eleven countries in the Region are among the 22 high-burden countries in the world. Most cases occur in the age group of 15-54 years, with males being disproportionately affected ${ }^{[2]}$. Bangladesh, with a population of over 150 million, is one of the five high burden countries for tuberculosis in the South-East Asia Region. The estimated prevalence of all forms of tuberculosis and incidence rate in the country was 391 and 225 per 100000 population, respectively in $2006^{[3]}$.

The causative agent of tuberculosis is Mycobacterium tuberculosis and it is a major public health concern, because the bacterium spreads from person to person, effective and above all rapid diagnosis is a key objective of worldwide tuberculosis control strategies. The common site of tuberculosis bacilli is the lungs, but now a day other organs have been observed to be affected by tuberculosis. During effective tuberculosis treatment most people with pulmonary tuberculosis get well but the patients with extra pulmonary tuberculosis (EPTB) remain undetectable mostly by conventional

Table 1 : Grading of AFB-microscopy

\begin{tabular}{ll}
\hline Number of Bacilli & Result reported \\
\hline No AFB per 100 oil immersion fields & Negative \\
$1-9$ AFB per 100 oil immersion fields & Seanty \\
$10-99$ AFB per 100 oil immersion fields & $+(1+)$ \\
$1-10$ AFB per oil immersion field & $++(2+)$ \\
$>10$ AFB per oil immersion field & $+1(3+)$ \\
\hline
\end{tabular}

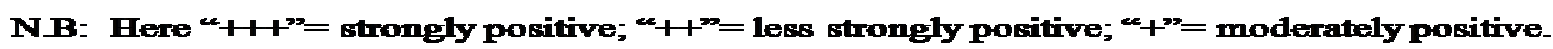


microscopy or other techniques ${ }^{[4]}$. Disseminated TB develops in the small number of infected people whose immune systems do not successfully contain the pulmonary infection.

Definitive diagnosis of tuberculosis involves demonstration of $M$. tuberculosis by microbiological, cytopathological or histopathological methods ${ }^{[5]}$. In a study where, Acid-Fast Bacilli (AFB) smears were found rarely positive with a detection rate of $<5 \%$ and culture detection of $M$. tuberculosis in pleural fluid ranged from 23 to $56 \%{ }^{[6]}$. Moreover $M$. tuberculosis grows slowly, which can lead to unacceptable delaying of diagnosis ${ }^{[7]}$. Though histology of extrapulmonary samples coupled with mycobacterial culture is thought to be most sensitive method currently available but could miss upto $20 \%$ of cases ${ }^{[6]}$. Clinical presentation of EPTB is atypical. Especially when the disease involves obscure occult sites, EPTB may not even be considered in the initial list of differential diagnosis. Further, invasive methods may have to be employed to secure tissue/body fluids for analysis. Many times representative tissue/body fluid may not be accessible. Even when adequate tissue is procured, the pathological findings may be suggestive of "granulomatous infection" which encompasses a wide range of differential diagnoses rather than "definitive tuberculosis". Therefore, the clinicians more often rely upon the clinical impression, radiological and endoscopic appearances and nonconventional diagnsotic methods as evidence to diagnose EPTB ${ }^{[5]}$.

Nucleic acid amplification (NAA) tests have emerged with the goal of enabling clinicians to make a rapid and accurate diagnosis. Polymerase chain reaction (PCR) is the best known NAA test ${ }^{[4]}$. It amplifies target nucleic acid regions that uniquely identify the Mycobacterium tuberculosis complex. Several nucleic-acid based amplification tests targeting various parts of the genome such as IS6110, 65kDa, TRC4, GCRS, etc. are available for the detection of Mycobacterium tuberculosis ${ }^{[8-14]}$.

An important advantage of NAA tests is the rapidity by which results can be obtained, about 3-6 hours from the receipt of specimen. Most of the PCR studies done on respiratory specimens showed that the sensitivity of PCR ranges from $74 \%$ to as high as $98 \%$ and specificity of $85 \%$ to as high as $100 \%{ }^{[15-17]}$. However, this method failed to show consistent results in extrapulmonary specimens, with sensitivity ranging from $45 \%$ to as high as $95 \%{ }^{[18-23]}$. As such, the amplification techniques do not yet have an established role in the diagnosis of extrapulmonary tuberculosis.

In the present investigation, a very sensitive but rapid technique, Real-Time PCR platform was used for successful diagnosis of patients with extra pulmonary tuberculosis and compared with the conventional AFB method.

\section{MATERIALS AND METHODS}

Study area and population

The complete study was conducted at Gastro Liver Hospital and Research Center situated at Dhaka, Bangladesh. Suspected patient referred to PCR for EPTB at the molecular diagnosis unit from January 2010 to March 2011 were enrolled in this study.

\section{Sample collection and processing}

Samples were collected from suspected patients from suspected parts of the body by clinicians. In this study a total of 99 samples were collected from 9 different parts of body including tissue, ascetic fluids, pleural fluids, cerebrospinal fluids (CSF), broncho alveolar lavage (BAL), tracheal aspirates, urine, Percutaneous nephrostomy (PCN) fluids and pus. Sample volume less than $3 \mathrm{ml}$ was not picked up for analysis except pus. After collection, the samples were diluted with equal volume of $4.0 \% \mathrm{NaOH}$ containing $0.5 \% \mathrm{~N}$-Acetyl-L-Cysteine and incubated at $37^{0} \mathrm{C}$ for 20 minutes. Then specimens were centrifuged at $10000 \mathrm{rpm}$ for 5 minutes. The resulting sediments were then resuspended and washed three times by phosphate-buffered saline (0.01 M; pH 6.8). The pellets were collected and used in the subsequent experiments such as AFBmicroscopy and RT-PCR.

Table 2: Compantive distribution of AFB Microscopy and Real-Time PCR results in different specimen.

\begin{tabular}{|c|c|c|c|c|c|c|c|c|}
\hline \multirow{2}{*}{ Specimen type } & \multirow{2}{*}{$\begin{array}{c}\text { No. of } \\
\text { Specimen }\end{array}$} & \multicolumn{5}{|c|}{ Microscopicresult } & \multicolumn{2}{|c|}{ PCR Result } \\
\hline & & negative & Scanty & $1+$ & $2+$ & 3+ & negative & Positive \\
\hline Tissue & 25 & 22 & 1 & 2 & $\mathbf{0}$ & $\mathbf{0}$ & 15 & 10 \\
\hline Ascitic Fluld & 21 & 18 & 2 & 1 & $\mathbf{0}$ & $\mathbf{0}$ & 12 & 9 \\
\hline Pleurel Fluld & 16 & 14 & 2 & $\mathbf{0}$ & $\mathbf{0}$ & $\mathbf{0}$ & 10 & 6 \\
\hline CSF & 14 & 14 & $\mathbf{0}$ & $\mathbf{0}$ & $\mathbf{0}$ & $\mathbf{0}$ & 11 & $\mathbf{3}$ \\
\hline $\mathbf{B A L}$ & 5 & 5 & $\mathbf{0}$ & $\mathbf{0}$ & $\mathbf{0}$ & $\mathbf{0}$ & 4 & 1 \\
\hline Tracheal Aspirates & 7 & 6 & $\mathbf{0}$ & 1 & $\mathbf{0}$ & $\mathbf{0}$ & 5 & 2 \\
\hline Urine & 7 & 7 & $\mathbf{0}$ & $\mathbf{0}$ & $\mathbf{0}$ & $\mathbf{0}$ & 6 & 1 \\
\hline PCN Fluid & $\mathbf{1}$ & 1 & $\mathbf{0}$ & $\mathbf{0}$ & $\mathbf{0}$ & $\mathbf{0}$ & 1 & 0 \\
\hline Pus & 3 & 2 & $\mathbf{0}$ & $\mathbf{0}$ & $\mathbf{0}$ & 1 & 2 & 1 \\
\hline Total & 99 & 89 & 5 & 4 & $\mathbf{0}$ & 1 & 66 & 33 \\
\hline
\end{tabular}




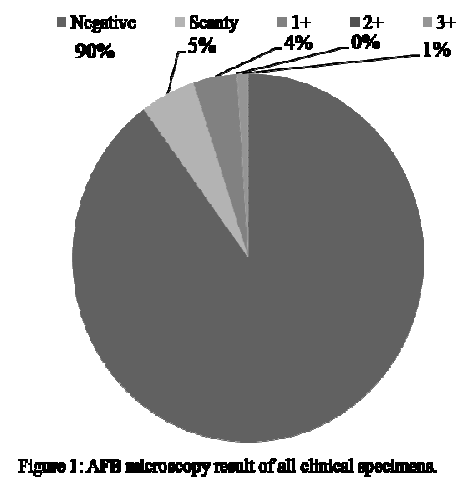

\section{AFB Microscopy}

Smears prepared from the pellets previously processed were heat fixed and stained using the hot Ziehl-Neelsen method (1\% carbol-fuchsin dye). Smears were scanned by an oil immersion microscope, before a smear was declared as negative at least 100 fields were examined. To conform the morphology of the bacilli all the smears were read using a magnification of $\times 1000$ and the results were recorded according to table 1 .

\section{DNA extraction}

DNA was extracted from $200 \mu \mathrm{l}$ of initially processed sample by the MTB Real-TM (Sacace Biotechnologies, IVD, Italy) following the manufacturer's instructions at the DNA Laboratory Unit of Gastro Liver Hospital and Research Center. DNA sample were stored at $4^{\circ} \mathrm{C}$ until Real-time PCR analysis was performed.

\section{Real-time PCR}

Real Time PCR was performed with primers FP-5'CCTGCGAGCGTAGGCGTCGG-3' and RP-5'-
CTCGTCCAGCGCCGCTTCGG-3' to amplify 123bp fragment of insertion element IS6110 of $M$. tuberculosis ${ }^{[24]}$ according the protocol described in MTB Real-TM. Master mixture was prepared following the instruction of manufacturer. TaqMan probe was used as fluorescent reporter dye specific for M. tuberculosis and M.tuberculosis IC. M. tuberculosis IC is DNA fragment of IS6110 insertion of Mycobacterium tuberculosis modified and cloned in bacteriophage $\lambda$, containing DNA fragments used in the kit as matrix for primers. Internal Control (IC) serves as an amplification control for each individually processed specimen and to identify possible reaction inhibition. IC is detected in a channel other than the $M$. tuberculosis DNA. Extracted and purified DNA was amplified by using Smart Cycler® (Germany) with the specific primer set. The amplification was done by $25 \mu 1$ of PCR mixture which was prepared by adding $15 \mu \mathrm{l}$ of master mixture $\{10 *(\mathrm{~N}+1) \quad \mu \mathrm{l}$ of PCR-mix-1, $5^{*}(\mathrm{~N}+1)$ of PCR Buffer Flu, $0,5^{*}(\mathrm{~N}+1)$ of TaqF DNA Polymerase and $0,5 *(\mathrm{~N}+1)$ of UDG-Enzyme, vortex and centrifuge briefly $\}$ and $10 \mu \mathrm{l}$ of DNA template. A negative control from DNA extraction and another two controls (Negative control and positive control) for each panel were also prepared for a successful PCR. In Smart Cycler® both amplification and detection were performed by the following thermal cycle- stage $1: 95^{\circ} \mathrm{C}$ for 900 seconds, stage $2: 95^{\circ} \mathrm{C}$ for 15 seconds, $65^{\circ} \mathrm{C}$ for 30 seconds and $72^{\circ} \mathrm{C}$ for 15 seconds for 5 repeated cycle and stage 3: $95^{\circ} \mathrm{C}$ for 15 seconds, $65^{\circ} \mathrm{C}$ for 30 seconds and $72^{\circ} \mathrm{C}$ for 15 seconds for 40 repeated cycle. Fluorescence is observed in Real Time on the FAM channel for M. tuberculosis DNA and on the $\mathrm{Cy} 3$ channel for Internal Control.

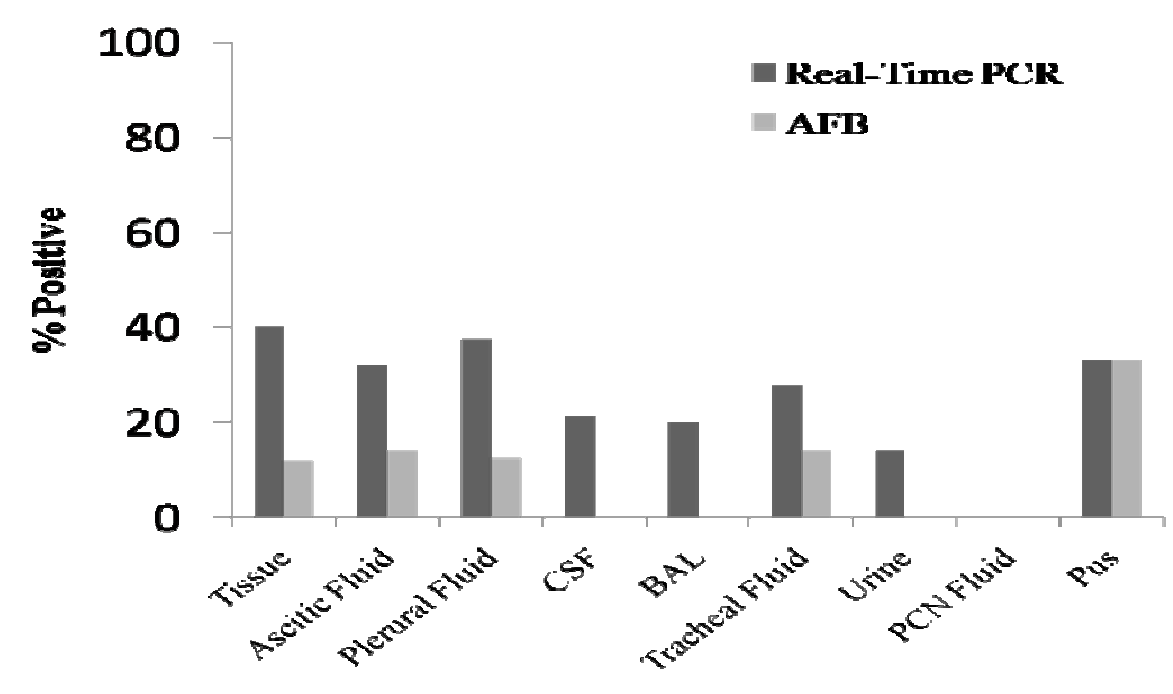

Specimens

Figure 2: Comparative results of Real-Time PCR and AFB microscopy in different specimens. 


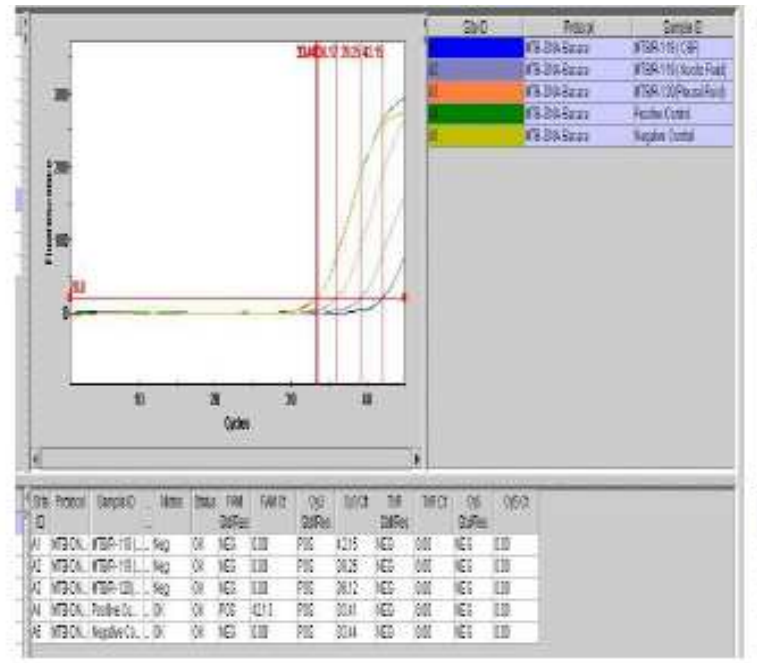

Figure:3a

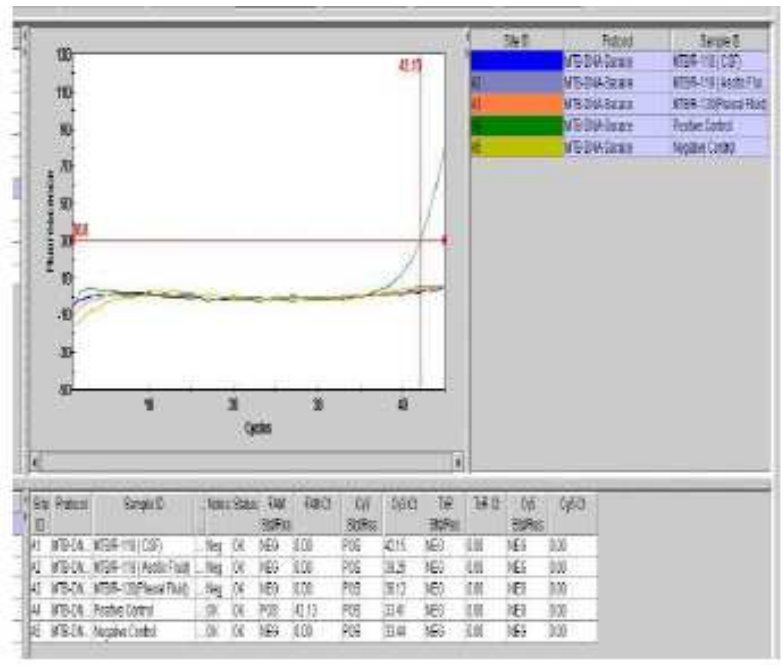

Figure:3b

Figure 3: A representative diagram of positive and negative samples in thermal cycler including the negative and positive control. Three samples (CSF, Ascitic fluid, Pleural fluid) with a positive control and negative control were run in a thermal cycler named Smart Cycler (Powered by Cepheid innovation. 3a: All the samples contained internal control (IC) which crossed the threshold level after different PCR cycle during amplification in Cy3 channel. Positive control crossed the threshold level at 42 cycles in FAM channel. 3b: Here all the samples showed negative result. Only positive control crossed the threshold.

\section{RESULTS AND DISCUSSION}

A total of ninety nine clinical specimens from suspected nine different extrapulmonary parts of the body were collected and processed for diagnosis of MTB complex (Table 2). All of the patients were highly suspected to be MTB positive by specialized clinicians according to their symptoms and complexities and there were no record of previous infection by MTB complex or any other species of Mycobacterium. None of them were taking antibiotic drugs against MTB as well. Out of 99 samples, $10(10.10 \%)$ samples were positive in AFB microscopy where $5.0 \%$ showed scanty positive, whereas $4.0 \%, 0 \%$ and $1.0 \%$ showed $1+, 2+$ and $3+$ respectively (Figure 1). Maximum $14.28 \%$ samples were positive in case of Ascitic fluid (3 out of 9) and Tracheal Aspirates (2 out of 7). Similar result was found in case of Pleural Fluid (12.5\%) and Tissue specimens $(12 \%)$ but no samples were found positive in CSF, BAL, Urine, PCN and Pus specimens (Figure 2).

For comparative analysis all the specimens were subjected to Real-Time PCR analysis. Out of 99 samples, 33 (33.3\%) (Table 2) were positive in RTPCR which is $23.33 \%$ more than the AFB method. Higher percentages of positive results were found in Real-Time PCR analysis in all specimens. Maximum $42.85 \%$ (9 out of 21) samples were positive in case of Ascitic fluid which is $28.57 \%$ more than the AFB method. Pleural Fluid (37.5\%), Tracheal Aspirates $(28.56 \%)$ and Tissue $(40 \%)$ samples also showed higher percentage of positive result than AFB microscopy. The samples which were positive in AFB microscopy were also positive and additional 23 samples were positive in RT-PCR (Figure 2). Though CSF, BAL and urine were completely negative in AFB microscopy but gave positive result in Real-Time PCR. PCN and Pus samples did not give any positive result in both methods but it's not conclusive in comparative analysis because sample numbers were too low for comparison.

The diagnosis of EPTB is made difficult by the complexity and risks involved in the collection of specimen for examination. It is challenging for a number of reasons: the lack of adequate sample amounts or volumes; the apportioning of the sample for various diagnostic tests (histology/cytology, biochemical analysis, microbiology, and PCR), resulting in nonuniform distribution of microorganisms; the paucibacillary nature of the specimens; the presence of inhibitors that undermine the performance of nucleic acid amplification-based techniques; and the lack of an efficient sample processing technique universally applicable on all types of extrapulmonary samples. This leaves clinicians with a long delay without an affirmative answer. But with the development of newer techniques in molecular biology, this delay in the accurate diagnosis of the disease is minimized.

In a study in Turkey by Ozkutuk evaluating the performance of Cobas Amplicor MTB (CAMTB) test for pulmonary and extrapulmonary specimens, results indicate that the CA-MTB is a rapid test for 
detection of tuberculosis in pulmonary specimens, but does not perform well enough in extrapulmonary specimens ${ }^{[25]}$. The poor performance of conventional microbiological techniques in extrapulmonary specimens has stimulated the increased use of PCR tests in the laboratory diagnosis of tuberculosis.

The exact diagnostic role of PCR assay for $M$. tuberculosis in high-prevalence areas for tuberculosis has to be assessed in appropriate control groups, particularly in the case of EPTB. Many studies were carried out in different part of the world showing the usefulness of PCR method targeting specific gene of M. tuberculosis ${ }^{[26,27]}$ but no definitive study among Bangladeshi population has been carried out before comparing the Real-Time PCR method with the conventional AFB microscopy. We showed here that PCR would be particularly useful for the diagnosis of EPTB by comparing with the conventional AFB microscopy among Bangladeshi population. In many studies the analysis was carried out in patients where the provisional diagnosis of tuberculosis was made on the basis of clinical presentation.

In case of AFB microscopy about $50 \%$ of samples were scanty positive and $40 \%$ were $1+$ positive which are considered as weak positive; on the other hand $0 \%$ and $10 \%$ samples were $2+$ and $3+$ positive which are considered as strong positive. So, AFB microscopy can't give us a comprehensive result for the diagnosis of TB. It is because, the sensitivity of microscopy depends on the clinical presentation and more than 10.000 bacilli per milliliter are necessary for secure microscopic positivity ${ }^{[28]}$.

Real-Time PCR is highly sensitive and widely accepted method for the diagnosis of any kind of infectious disease. The primer used in this study is very specific for Mycobacterium tuberculosis. Though the presence of fewer copies of target sequence IS6110, in some strains of M. tuberculosis has been reported ${ }^{[29,30]}$, but it has been also reported in some earlier studies that PCR assay targeting IS6110 were more sensitive ${ }^{[31,32]}$ and more useful compared to other targets like $65 \mathrm{kDa}, 38 \mathrm{kDa}$, and $85 \mathrm{~B}$ protein ${ }^{[33]}$. The methodology of PCR for IS6110 has been also carried out in different technical setups and has been proven to be simple and reproducible, compared to methodologies for PCR targeting other gene sequences. In this study the accuracy of primers was confirmed by using positive control (Fig 3a and 3b) which crossed the threshold level after certain cycles of amplification. In this study higher percentage of positive results were found when RealTime PCR analysis was carried out in all specimens except PCN Fluid and Pus, where AFB and RealTime PCR showed same results. In both these specimens, sample numbers were 1 and 3 respectively which are too low for any kind of comparative analysis. A sharp difference is visible between the two methods where the sample numbers are sufficiently large for comparison. Analysis of more samples would produce more comprehensive difference between these two methods.
Though it is reported in earlier studies about the usefulness and sensitivity of IS6110 over AFB microscopy and culture method using conventional PCR method ${ }^{[31,32]}$ but Real-Time PCR could be much more useful in terms of rapidity of producing perfect result. The rapidity, high sensitivity and simplicity of PCR targeting IS6110 gene sequence can compensate the higher cost of the test compared with less sensitive conventional tests in the diagnosis of extrapulmonary tuberculosis and facilitate the therapeutic decision made by the clinician.

\section{ACKNOWLEDGEMENTS}

We thank Gastroliver hospital and research center for providing laboratory and financial support for this study.

\section{REFERENCES}

1. WHO Report (2001). Global tuberculosis control. Geneva: World Health Organization, 2001:18-19.

2. The regional report (2008). TB in South-East Asian Region (SEAR). World Health Organizaion:2008.p3

3. The regional report (2008). TB in South-East Asian Region (SEAR). World Health Organizaion:2008.p25

4. American Thoracic Society Workshop (1997). Rapid diagnostic tests for tuberculosis. What is the appropriate use? Am J Respir Crit Care Med. 155: 1804-1814.

5. Sharma SK and Mohan A (2004). Extrapulmonary tuberculosis. Indian J Med Res 120: 316-353.

6. Trajman A, Pai M, Dheda K, Van Zyl Smit A and Zwerling AA (2008). Novel tests for diagnosis tuberculosis pleural effusion: What works and what does not? Eur. Respiratory J. 31: 1098-1106.

7. Colmenero JD, Jiménez-Mejı́as ME, Reguera JM, Palomino-Nica's J, Ruiz- Mesa JD, MárquezRivas J, Lozano A and Pachón J (2004) Tuberculous vertebral osteomyelitis in the new millennium: still a diagnostic and therapeutic challenge. Eur J Clin Microbiol Infect Dis. 23: 477-483.

8. de Lassence A, Lecossier D, Pierre C, Cadranel J, Stern M and Hance AJ (1992). Detection of mycobacterial DNA in pleural fluid by means of the polymerase chain reaction: comparison of two protocols. Thorax. 47:265-269.

9. de Wit D, Maartens G and Stein L (1992). A comparative study of the polymerase chain reaction and conventional procedures for the diagnosis of tuberculous pleural effusion. Tubercle Lung Dis. 73:262-267.

10. Nagesh BS, Sehgal S, Jindal SK and Arora SK (2001). Evaluation of polymerase chain reaction 
for the detection of Mycobacterium tuberculosis in pleural fluid. Chest. 119:1737-1741.

11. Parandaman V, Narayanan and Narayanan PR (2000). Utility of polymerase chain reaction using two probes for rapid diagnosis of tubercular pleuritis in comparison to conventional methods. Indian J. Med. Res. 112: 47-51.

12. Querol J, Minguez J, Garcia-Sanchez E, Farga MA, Gimeno C and Garcia-de-Lomas J (1995). Rapid diagnosis of pleural tuberculosis by polymerase chain reaction. Am. J. Respir. Crit. Care Med. 52:1977-1981.

13. Verma A, Dasgupta N, Agarwal AN, Pande JN and Tyagi JS (1995). Utility of a Mycobacterium tuberculosis GC-rich repetitive sequence in the diagnosis of tuberculous pleural effusion by PCR. Indian J. Biochem. Biophys. 32:429-436.

14. Villena M, Robollo J, Aguada JM, Galan A, Encuentra L and Pulenque E (1998). Polymerase chain reaction for the diagnosis of pleural tuberculosis in immunocompromised and immunocompetent patients. Clin. Infect. Dis. 26:212-214.

15. Cheng VC, Yam WC and Hung IF (2004) Clinical evaluation of the polymerase chain reaction for the rapid diagnosis of tuberculosis. $J$ Clin Pathol. 57:281-285.

16. Ashok R (2000). PCR for the diagnosis of tuberculosis: Where are we now? Indian Journal of Tuberculosis. 47:79-82.

17. Bergmann JS and Woods GL (1996). Clinical evaluation of the Roche AMPLICOR PCR Mycobacterium tuberculosis test for detection of M. tuberculosis in respiratory specimens. J Clin Microbiol. 34:1083-1085.

18. Shah S, Miller A and Mastellone A (1998). Rapid Diagnosis of Tuberculosis in various biopsy and body fluid specimens by the Amplicor Mycobacterium tuberculosis PCR test. Chest.113:1190-1194.

19. Scarparo C, Piccolo P and Rigon A (2000). Comparison of Enhanced MTB Amplified Direct Test with Cobas Amplicor Mycobacterium tuberculosis Assay for Direct Detection of $\mathrm{M}$. tuberculosis complex in respiratory and extrapulmonary specimens. J Clin Microbiol. 38: 1559-1662.

20. D'Amato R, Hochstein $\mathrm{L}$ and Colaninno $\mathrm{P}$ (1996). Application of Roche Amplicor Mycobacterium tuberculosis PCR test to specimens other than respiratory secretions. Diagn Microbiol Infect Dis. 24:15-17.

21. Piersimoni C, Scarparo C, Piccolo P and Rigon A (2002). Performance Assessment of Two Commercial Amplification Assay for Direct Detection of $\mathrm{M}$. tuberculosis complex for respiratory and extrapulmonary specimens. J Clin Microbiol. 40:4138-4142.

22. Yee Y, Gough A and Kumarasinghe G (2002). The pattern and utilization of accuracy of a commercial NAA test for the rapid diagnosis of M. tuberculosis in routine clinical practice. Singapore Med J. 43:415-420.

23. Eisenach KD, Cave MD, Bates JH and Crawford JT (1990). Polymerase chain reaction amplification of repetitive DNA sequence specific for Mycobacterium tuberculosis. J infect Dis. 161: 977-981.

24. Ozkutuk A, Kirdar S and Ozden S (2006). Evaluation of Cobas Amplicor M. tuberculosis test to detect $M$. tuberculosis in pulmonary and extrapulmonqary specimens. New Microbiol. 29:269-273.

25. Sekar B, Selvaraj L, Alexis A, Ravi S, Arunagiri $\mathrm{K}$ and Rathinavel L (2008). The utility of IS6110 sequence based polymerase chain reaction in comparison to conventional methods in the diagnosis of extra-pulmonary tuberculosis. Indian J Med Microbiol. 26:352-355.

26. Maurya AK, Kant S, Nag VL, Kushwaha RAS, Kumar M and Dhole TN (2011). Comparative evaluation of IS6110 PCR via conventional methods in rapid diagnosis of new and previously treated cases of extrapulmonary tuberculosis. Tüberküloz ve Toraks Dergisi. 59(3): 213-220.

27. Carpentier E, Drouillard B, Dailloux M, Moinard D, Vallee E and Dutilh B (1995). Diagnosis of tuberculosis by Amplicor Mycobacterium tuberculosis test: a multicenter study. J Clin Microbiol. 33: 3106-10.

28. Agasino CB, Ponce de Leon A, Jasmer RM and Small PM (1998). Epidemiology of Mycobacterium tuberculosis strains in San Francisco that do not contain IS6110. Int J Tuberc Lung Dis. 2:518-520.

29. Das S, Paramasivan CN, Lowrie DB, Prabhakar R and Narayanan PR (1995). IS6110 restriction fragment length polymorphism typing of clinical isolates of Mycobacterium tuberculosis from patientswith pulmonary tuberculosis in Madras, South India. Tuberc Lung Dis. 76:550-554.

30. Tiwari V, Jain A and Verma RK (2003). Application of enzyme amplified mycobacterial DNA detection in the diagnosis of pulmonary and extrapulmonary tuberculosis. Indian $\mathrm{J}$ Med Res. 118:224-228.

31. Kesarwani RC, Pandey A, Misra A and Singh AK (2004). Polymerase chain reaction (PCR): Its comparison with conventional techniques for the diagnosis of extra-pulmonary tubercular diseases. Indian J surgery. 66:84-88.

32. Negi SS, Anand R, Pasha ST, Gupta S, Blasir SF, Khare S and Lal S (2007). Diagnostic potential of 
IS6110, $38 \mathrm{kDa}$ and $85 \mathrm{~B}$ sequence based polymerase chain reaction in the diagnosis of
Mycobacterium tuberculosis in clinical samples.

Indian J Med Microbiol. 25:43-49. 